\title{
Effect of Partial Water Saturation on Attenuation Characteristics of Low Porosity Rocks
}

\author{
Tae-Min Oh • Tae-Hyuk Kwon • Gye-Chun Cho
}

Received: 25 August 2010/Accepted: 4 October 2010/Published online: 21 October 2010

(C) The Author(s) 2010. This article is published with open access at Springerlink.com

Keywords Wave attenuation · Partial water saturation · Compressional wave $\cdot$ Longitudinal wave $\cdot$ Low porosity rock

\section{Introduction}

With the advance of geophysical exploration techniques, in situ measurements on elastic wave velocities have been successfully employed for investigating near-surface and deep geological structures. However, in situ techniques that measure the attenuation of elastic waves are still in their infancy due to the difficulty of calibration, the lack of theoretical models, and the inaccuracy of field scale measurements. While attenuation itself is strongly related to important physical characteristics of porous media, such as pore fluid composition, stress states, and internal heterogeneity (Johnston et al. 1979), the understanding of the attenuation characteristics of porous media is still limited.

The rocks are frequently exposed to water through rain or ground water. The presence of pore water not only plays

T.-M. Oh · G.-C. Cho $(\bowtie)$

Department of Civil and Environmental Engineering,

Korea Advanced Institute of Science and Technology (KAIST),

Taejon 305-701, Korea

e-mail: gyechun@kaist.edu

T.-M. Oh

e-mail: ohtaemin@kaist.ac.kr

T.-H. Kwon

Earth Sciences Division, Lawrence Berkeley National

Laboratory, 1 Cyclotron Rd. MS 90R1116,

Berkeley CA 94720, USA

e-mail: thkwon@lbl.gov an important role from an engineering perspective, but also significantly alters acoustic wave propagation. Compressional wave velocity changes little until the pore spaces are fully saturated with water because the air in partially saturated pore fluids diminishes the stiffness of the pore fluids and hardly contributes to strengthening the rock frame. On the other hand, when there is an increase in water saturation, the compressional wave attenuation - that is related to energy dissipation - tends to increase more sensitively than velocity (Gardner et al. 1964; Toksőz et al. 1979; Mavko and Nur 1979; Murphy 1982; Winkler and Nur 1982; Cadoret et al. 1998).

Several previous researchers have investigated the fluid effect on the wave velocity and attenuation, mainly focusing on high porosity rocks (mostly greater than $20 \%$ of porosity), such as limestone (Cadoret et al. 1998) and sandstone (Murphy 1982; Winkler and Nur 1982). This study, therefore, examines how water saturation affects the attenuation characteristics of low porosity rocks. The effect of partial water saturation on the attenuation is explored by recapitulating Biot model and by conducting a series of laboratory tests. Then, the results of Biot model and laboratory tests are compared and some implications are discussed.

\section{Recapitulation of Biot Model: Partial Water Saturation Effect on Attenuation}

Biot model (Biot 1956a, b) can be applied to porous media, particularly for modeling wave velocity and attenuation of a rock. Biot model assumes that pore fluids flow only in the direction parallel to the direction of the wave propagation and that the viscous fluid motion follows Poiseuille flow in a cylindrical tube. No chemical, electrical, or thermal 
interactions between different phases are taken into account in the model. Detailed formulations of the Biot model and the physical parameters used are summarized in Appendix A. The following parametric study recapitulates Biot model and explores how various physical properties of rocks, such as porosity, permeability, or pore size parameter, affect the attenuation of compressional wave (hereafter, P-wave) in a partially water-saturated porous medium. The pore fluid properties (e.g., bulk modulus and density), in which the composition of water and air vary, are calculated by the averaging method (more details are described in the Appendix section).

\subsection{Porosity}

Attenuation increases as the porosity increases because the water content in a pore becomes larger at the same degree of water saturation. Higher porosity rocks can have more opportunity of interaction between fluid and rock frame in the cracks than lower porosity rocks. Specifically, Fig. 1a shows that the attenuation of a high porosity rock (when $\left.n=30 \%, Q^{-1}=0.0046\right)$ is approximately nine times larger than that of a low porosity rock (when $n=1 \%$, $\left.Q^{-1}=0.0005\right)$ at a saturation degree of $98-99 \%$, which is the saturation degree required for a maximum attenuation value.

\subsection{Permeability}

For low permeability rocks, the pressure gradient induced by wave propagation dissipates slowly and there are minimal flows of fluid in the pores and pore throats. Meanwhile, for higher permeability rocks, fluids can move more freely, and consequently cause greater loss of energy during the wave propagation. It is found that permeability has the strongest impact on the magnitude of the attenuation than porosity and pore size, affecting approximately one order of magnitude increase of attenuation by an order of magnitude increase of the permeability, as shown in Fig. $1 b$.

\subsection{Pore Size Parameter}

The pore size parameter is an indicator of the geometric characteristics of the pore space. As shown in Fig. 1c, when the rock has the same porosity and permeability, the attenuation increases as the pore size parameter decreases. It implies that the wave loses more energy in smaller-sized pores at the same porosity.

\subsection{Attenuation Curve Tendency}

The attenuation $\left(Q^{-1}\right)$ is normalized by the maximum attenuation $\left(Q_{\max }^{-1}\right)$, which is the attenuation value for
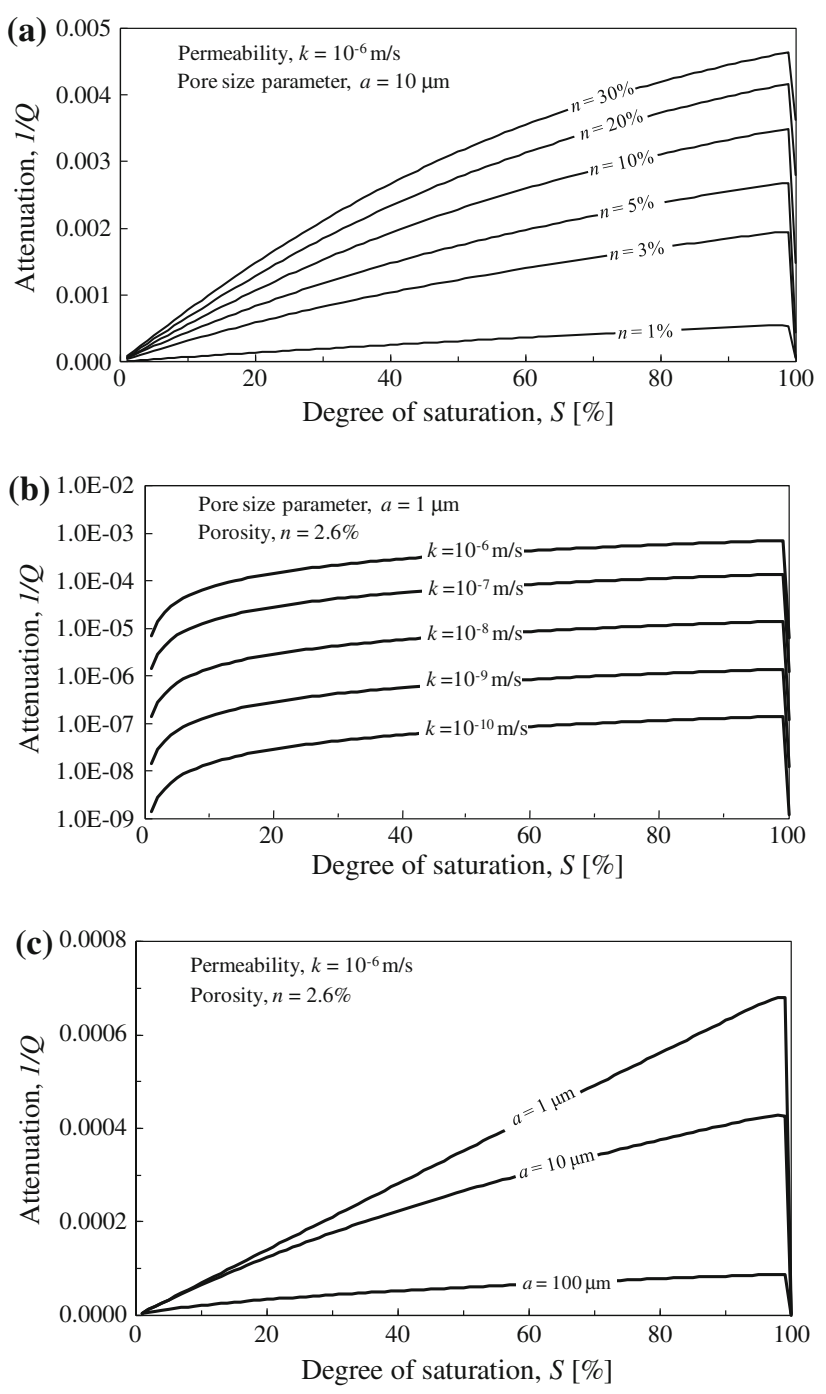

Fig. 1 Attenuation versus water saturation varying a porosity, b permeability, and c pore size parameter when the Young's modulus $E=45 \mathrm{GPa}$, frequency $f=23 \mathrm{kHz}$, and Poisson's ratio $v=0.24$

nearly full water-saturated conditions (i.e., $S \approx 99 \%$ ). The normalized value enables us to examine how the curve tendency and shape depend on the various parameters of low porosity rocks. The attenuation-water saturation $\left(Q^{-1} /\right.$ $Q_{\max }^{-1}-S$ ) curves (hereafter, attenuation curve) are superimposed in Fig. 2 to highlight the effect of changes in the permeability and pore size parameter. Porosity is excluded as a variable because it has a negligible effect on the attenuation curve tendency. Figure 2 shows that the attenuation curve shape with water saturation can be convex, linear, or concave depending on the permeability. A high permeability $\left(k \geq 10^{-5} \mathrm{~m} / \mathrm{s}\right.$ in the presented study) produces a concave attenuation curve. In contrast, the attenuation curve is convex when the permeability is lower than $10^{-5} \mathrm{~m} / \mathrm{s}$. It is worth to note that a small pore size 


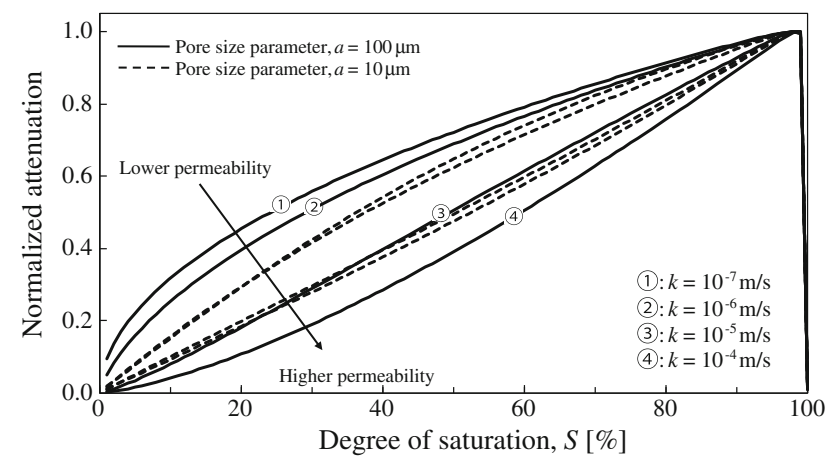

Fig. 2 Normalized attenuation curves $\left(Q^{-1} / Q_{\max }^{-1}\right)$ with respect to a range of the permeabilities and pore sizes when the porosity $n=2.6 \%$, Young's modulus $E=45 \mathrm{GPa}$, frequency $f=23 \mathrm{kHz}$, and Poisson's ratio $v=0.24$

parameter produces a linear curve: the attenuation curve is almost linear with water saturation when the pore size parameter is less than $1 \mu \mathrm{m}$ (also refer to Fig. 1c).

\section{Experimental Program}

\subsection{Specimen Preparation}

Four cylindrical rock specimens were prepared, representing unique low porosity characteristics of deep bedrock formations in Korea: two weathered granite and two mudstone specimens. All the specimens had a diameter of $5 \mathrm{~cm}$, a length of $10 \mathrm{~cm}$, and a porosity value of less than $3 \%$. The dry density of the specimens was measured after drying them in an oven at $50^{\circ} \mathrm{C}$ for $72 \mathrm{~h}$, while the water-saturated density was measured after submerging them until the measured masses converged to a certain value. The effective porosity, which is a measure of connected pores, but not closed pores, was estimated from a dry density and a fully water-saturated density. A standard method suggested by the International Society for Rock Mechanics (ISRM; Brown 1981) was used to determine the specific gravity of each specimen. The properties of the specimens tested are summarized in Table 1.

\subsection{Saturating and Drying Processes}

To determine water content, porosity, density, absorption, and related properties, saturating and drying processes were performed by following the procedures suggested by ISRM (Brown 1981). A saturating process was performed by completely immersing a specimen in a water bath. The water bath was agitated to remove any trapped air. After a saturating procedure, the specimen was dried in an oven at a constant temperature of $50^{\circ} \mathrm{C}$ in order to prevent any
Table 1 Properties of tested specimens

\begin{tabular}{|c|c|c|c|c|}
\hline Specimen & Granite 1 & Granite 2 & Mudstone 1 & Mudstone 2 \\
\hline Symbol & GN1 & GN2 & MS1 & MS2 \\
\hline \multicolumn{5}{|l|}{ Density $\left(\mathrm{kg} / \mathrm{m}^{3}\right)$} \\
\hline Dry & 2,591 & 2,596 & 2,628 & 2,626 \\
\hline Saturation & 2,603 & 2,609 & 2,637 & 2,652 \\
\hline Porosity (\%) & 1.16 & 1.26 & 0.96 & 2.61 \\
\hline Specific gravity (-) & 2.62 & 2.62 & 2.65 & 2.64 \\
\hline $\begin{array}{l}\text { Rod-wave velocity } \\
(\mathrm{m} / \mathrm{s})^{\mathrm{a}}\end{array}$ & 1,864 & 1,105 & 4,891 & 4,137 \\
\hline $\mathrm{P}$-wave velocity $(\mathrm{m} / \mathrm{s})^{\mathrm{b}}$ & 2,619 & 1,625 & 5,273 & 4,459 \\
\hline Young's modulus $(\mathrm{GPa})^{\mathrm{c}}$ & 9.4 & 3.3 & 62.9 & 45 \\
\hline Poisson's ratio $(-)^{\mathrm{d}}$ & 0.38 & 0.39 & 0.24 & 0.24 \\
\hline
\end{tabular}

${ }^{\text {a }}$ Rod-wave velocity of a dry specimen at the resonance frequency was determined by using a free-free resonant column (FFRC) method (Vaghela and Stokoe 1995; Kim et al. 1997; Cha and Cho 2007)

${ }^{b}$ P-wave velocity of a dry specimen was determined by using the pointsource travel time method (Cha and Cho 2007)

c Young's modulus (i.e., $E=\rho V_{\mathrm{P}}^{2}$ ) was calculated by using the dry density and the P-wave velocity of a specimen at a dry condition

' Poisson's ratio is calculated by using the P-wave velocity and rod-wave velocity of a specimen at a dry condition

damage induced by thermal shock. As time elapses, the degree of water saturation was periodically evaluated by measuring the mass, and the attenuation was simultaneously measured.

\subsection{Free-Free Resonant Column Test}

The attenuation of a rock specimen was obtained by a free-free resonant column (FFRC) test (Vaghela and Stokoe 1995; Kim et al. 1997; Cha and Cho 2007). The FFRC method measures the attenuation of the longitudinal wave mode (i.e., also called as rod wave or bar wave). A typical result of the FFRC test at the time domain is shown in Fig. 3a. The damping ratio $(D)$ is obtained by fitting the frequency response curve of a single degree of freedom system to the measured data as shown in Fig. $3 \mathrm{~b}$. The attenuation is expressed in terms of the inverse quality factor $\left(Q^{-1}\right)$ calculated by the relationship, $Q^{-1}=2 D$.

Attenuation is highly affected by frequency and confining pressure. All the measurements were taken under atmospheric pressure and a room temperature of $25^{\circ} \mathrm{C}$. The FFRC measurements were conducted within the regime of the resonant frequency between 6 and $27.5 \mathrm{kHz}$. The signals were captured regularly until the measured mass of the specimen converged to a certain value, where the specimen was considered as fully water-saturated. We subsequently subjected the saturated specimens to a drying process in an oven and repeated the same elastic wave and mass measurement procedure. 

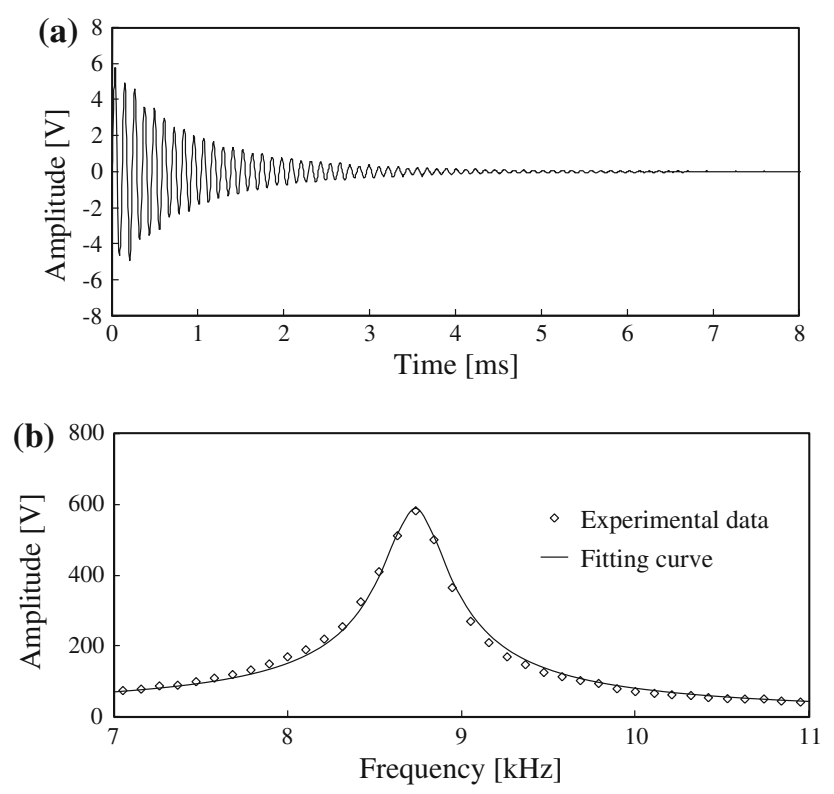

Fig. 3 A typical signal measured by an FFRC test at a the time domain and $\mathbf{b}$ the frequency domain

\section{Results and Discussion}

Overall, the experimental results deviate far from Biot model's prediction, showing that the experimental attenuation results (Fig. 4) are approximately orders of magnitude greater than Biot attenuation results (Fig. 1). This underestimation may be due to the fact that Biot model does not take random micro-cracks which are closely related to permeability into consideration.

\subsection{Attenuation Under a Dry Condition}

According to Biot model, the wave does not dissipate under a dry condition (i.e., $0 \%$ saturation); hence, the attenuation is expected to be null ( $Q^{-1} \rightarrow 0$ when $S \rightarrow 0$ ). However,

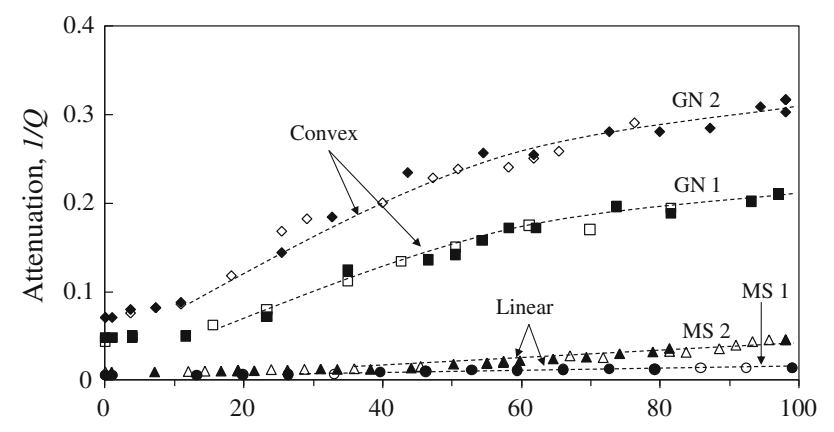

Fig. 4 Attenuations of longitudinal waves at difference water saturations. Note that the open points indicate the measurements during drying process whereas the solid points indicate the measurements during saturating process attenuations are unexceptionally measured for natural rock specimens under a dry condition (e.g., GN1: 0.048, GN2: 0.071, MS1: 0.006, and MS2: 0.010). These experimental measurements represent pure material damping without fluid effect, where the material damping is decided by frictional energy loss between cracks (or mineral grain boundaries) during elastic wave propagation (Winkler and Nur 1982). As shown in Fig. 4, granite specimens have higher attenuations under a dry condition than the mudstone specimens do because the mudstone specimens are much stiffer and have a smaller particle size than the granite specimens. It indicates that the energy loss due to internal heterogeneity-induced wave scattering, such as micro-cracks, is greater in the granite specimens than in the mudstone specimens.

\subsection{Attenuation Under Partial Water Saturation}

Attenuation curve tendency can be considered to have two distinctive regimes: low saturation regime and mid-to-high saturation regime. In the low saturation regime, the attenuation behavior as a function of saturation is dominated by the microscopic fluid flow mechanism (e.g., as explained by the squirt flow model; Cadoret et al. 1998). On the other hand, attenuation behavior in the mid-to-high saturation range is governed by a macroscopic mechanism (e.g., as explained by Biot model).

In a low water saturation regime, as shown in Fig. 4, attenuation of the low porosity rocks hardly or slightly increases with water saturation. This region covers the saturation from 0 to approximately 10 or $20 \%$. In contrast, in high-porosity rocks (e.g., Murphy 1982; Cadoret et al. 1998), the region that provides no or slight increase in attenuation with increasing water saturation covers a wider range of water saturation, i.e., from 0 to approximately 60 or $70 \%$. This phenomenon is due to the fact that the high porosity rock requires more water to reveal Biot effect, which causes water-solid coupling and decoupling.

In the mid-to-high water saturation regime, Biot model can be applicable for predicting the attenuation tendency and comparing it to measurements because Biot model is based on a macroscopic mechanism. Typical attenuationwater saturation curves are shaped as convex, linear, or concave (see Fig. 2). The attenuation curves of the granite specimens are convex, while the curves of the mudstone specimens are linear in the mid-to-high water saturation regime, as shown in Fig. 4. The linearity of the attenuation curves of the mudstone specimens is attributed to the small pore size of the mudstone specimen. In contrast to low porosity rocks, it has been presented in previous studies (e.g., Murphy 1982; Cadoret et al. 1998) that the attenuation curves of high-porosity rocks $(n>23 \%)$ are presumed to be concave in the mid-to-high saturation regime. This 
Table 2 Measured wave velocities and attenuations of the tested specimens

\begin{tabular}{|c|c|c|c|c|c|c|c|}
\hline \multirow[t]{2}{*}{ Specimen } & \multirow[t]{2}{*}{ Resonant frequency $(\mathrm{kHz})$} & \multicolumn{2}{|c|}{ P-wave velocity $(\mathrm{m} / \mathrm{s})$} & \multicolumn{2}{|c|}{ Rod-wave velocity (m/s) } & \multicolumn{2}{|c|}{ Attenuation $(1 / Q)(-)$} \\
\hline & & Dry & Saturated & Dry & Saturated & Dry (Min.) & Saturated (Max.) \\
\hline Granite 1 (GN1) & 9.5 & 2,619 & 2,794 & 1,864 & 1,906 & 0.048 & 0.210 \\
\hline Granite 2 (GN2) & 6.0 & 1,625 & 1,820 & 1,105 & 1,275 & 0.071 & 0.316 \\
\hline Mudstone 1 (MS1) & 27.5 & 5,273 & 5,318 & 4,891 & 4,966 & 0.006 & 0.014 \\
\hline Mudstone 2 (MS2) & 23.0 & 4,459 & 4,508 & 4,137 & 4,175 & 0.010 & 0.046 \\
\hline
\end{tabular}

concave shape of the attenuation curve in high-porosity rocks is consistent with the calculations in Biot model, as shown in Fig. 2 (i.e., when permeability $k$ is larger than $10^{-5} \mathrm{~m} / \mathrm{s}$ and the pore size parameter $a$ is larger than $10 \mu \mathrm{m})$.

A hysterical loop of attenuation during the saturating and drying processes is generally evident in tests on high porosity rocks due to different water distribution conditions (Cadoret et al. 1998). In contrast, the fluid distribution during the saturating and drying processes of low porosity rock has little effect on the attenuation. Figure 4 shows that the attenuation values during the saturation process are almost the same as those of the drying process for low porosity rocks.

\subsection{Attenuation Under a Fully Water-Saturated Condition}

The attenuation values of the tested specimens when fully saturated are nearly two to five times greater than the values under a dry condition as shown in Table 2 and Fig. 4. In particular, Fig. 4 indicates that the granite specimens are strongly influenced by a saturation level. GN2 (the softest rock in the presented study) has larger difference in attenuation between dry and saturation conditions than does MS1 (the hardest rock in the presented study). This means that a soft rock (e.g., a rock with low stiffness and slow wave velocity) is easily affected by fluid flow during saturation.

It is worth noting that high porosity rocks demonstrate attenuation drops in a fully water saturated condition (refer to the data in Murphy 1982; Cadoret et al. 1998). This drop is because no patchy water distribution and no squirt flow occur when water fully saturates the pores in a rock; as a result, the pore fluid cannot easily squeeze or flow into adjacent pores. Thus, the energy loss decreases. However, this phenomenon is not observed in the low porosity rock specimens tested (refer to Fig. 4). This result implies that low porosity rocks are unlikely to be fully water-saturated under a natural atmospheric environment without some additional pressure due to water surface tension on the micro-cracks and closed pores, both of which form during rock diagenesis. This condition is expected in various nearsurface grounds.

\section{Conclusions}

This study explored how water saturation affects the compressional wave attenuation characteristics in low porosity rocks by reviewing Biot model and performing a series of experiments. Main findings are as follows:

- Biot model shows that permeability is the most influential factor on attenuation due to wave energy dissipation by fluid flow between cracks (or pores).

- The experimental results deviate far from Biot model's prediction, showing that the experimental attenuation results are approximately orders of magnitude greater than Biot attenuation results. This discrepancy (i.e., Biot model's underestimation) may be due to the fact that Biot model does not take into consideration random micro-cracks which are closely related to permeability.

- The curve of attenuation versus saturation can be convex, linear, or concave from low to high permeability. The experimental results show that the attenuation of a low porosity rock tends to be convex or linear and shows no hysterical loop during the saturating and drying process.

- In the low saturation regime, the attenuation of low porosity rocks is dominated by the microscopic fluid flow mechanism (e.g., Squirt flow model) while in the mid-to-high saturation range, it is governed by a macroscopic mechanism (e.g., Biot model).

Acknowledgments This work was supported by the Smart InfraStructures Technology Center (SISTeC) under KOSEF and the grant (07UrbanRenaissanceB03) from High-Tech Urban Development Program funded by the Ministry of Construction \& Transportation of Korean Government.

Open Access This article is distributed under the terms of the Creative Commons Attribution Noncommercial License which permits any noncommercial use, distribution, and reproduction in any medium, provided the original author(s) and source are credited. 
Table 3 Parameters used in this study

\begin{tabular}{|c|c|c|}
\hline Parameter & Definition & Values selected for this study \\
\hline$a$ & Pore size parameter & \\
\hline$B_{\mathrm{a}}$ & Air bulk modulus & $142 \mathrm{kPa}$ at $1 \mathrm{~atm}^{\mathrm{a}}$ \\
\hline$B_{\mathrm{f}}$ & Fluid bulk modulus & $B_{\mathrm{f}}=\frac{1}{\frac{S}{B_{\mathrm{W}}}-\frac{1-S}{B_{\mathrm{a}}}}$ \\
\hline$B_{\mathrm{g}}$ & $\begin{array}{l}\text { Grain (particle) bulk } \\
\text { modulus }\end{array}$ & $50 \mathrm{GPa}^{\mathrm{a}}$ \\
\hline$B_{\text {sk }}$ & Skeleton bulk modulus & $B_{\mathrm{sk}}=E / 3(1-2 v)$ \\
\hline$B_{\mathrm{w}}$ & Water bulk modulus & $2.18 \mathrm{GPa}$ at $1 \mathrm{~atm}^{\mathrm{a}}$ \\
\hline$E$ & Elastic modulus & Dynamic Young's modulus \\
\hline$G_{\mathrm{sk}}$ & Skeleton shear modulus & $G_{\mathrm{sk}}=E / 2(1+v)$ \\
\hline$k$ & Permeability & \\
\hline$K$ & $\begin{array}{l}\text { Absolute hydraulic } \\
\text { conductivity }\end{array}$ & $\begin{array}{l}K=\eta \cdot k /\left(g \cdot \rho_{\mathrm{f}}\right) \text { where } g \text { is } \\
\text { gravitational acceleration } \\
\left(g=9.8 \mathrm{~m} / \mathrm{s}^{2}\right)\end{array}$ \\
\hline$n$ & Porosity & \\
\hline$S$ & Degree of saturation & \\
\hline$T$ & Visco-dynamic operator & $\begin{array}{l}T=\mathrm{e}^{(3 / 4) j \pi}\left(J_{1}\left(\zeta \cdot \mathrm{e}^{-j \cdot \pi / 4}\right) /\right. \\
\left.J_{0}\left(\zeta \cdot \mathrm{e}^{-j \cdot \pi / 4}\right)\right)^{\mathrm{b}} \\
J_{1} \text { and } J_{0} \text { are Bessel functions } \\
\text { and } j^{2}=-1\end{array}$ \\
\hline$\alpha$ & Tortuosity factor & $\begin{array}{l}\alpha=0.8(1-n)+1^{\mathrm{c}} \\
\alpha=1 \text { for tubes, } \alpha=2-3 \text { for } \\
\text { particulate materials }\end{array}$ \\
\hline$\rho$ & Mass density & $\begin{array}{l}\rho_{\mathrm{g}}=2,650 \mathrm{~kg} / \mathrm{m}^{3} \text { of } \\
\text { grain }(\text { particle }) \text { density } \\
\rho_{\mathrm{w}}=1,000 \mathrm{~kg} / \mathrm{m}^{3} \text { of water } \\
\rho_{\mathrm{f}}=S \rho_{\mathrm{w}} \text { of pore fluid } \\
\rho_{\text {mix }}=(1-n) \rho_{\mathrm{g}}+n \cdot S \cdot \rho_{\mathrm{w}} \\
\text { of mixture composed of } \\
\text { porous medium with fluid }\end{array}$ \\
\hline$\eta$ & Dynamic viscosity & $0.001 \mathrm{~Pa} \mathrm{~s}$ at $20^{\circ} \mathrm{C}$ for water \\
\hline$\zeta$ & Dimensionless factor & $\zeta=a\left(\omega \cdot \rho_{\mathrm{f}} / \eta\right)^{0.5 \mathrm{~b}}$ \\
\hline$v$ & Poisson's ratio & \\
\hline$\omega$ & Angular frequency & $\begin{array}{l}\omega=2 \pi f \text { (used resonant } \\
\text { frequency for } f \text { ) }\end{array}$ \\
\hline
\end{tabular}

\footnotetext{
a Santamarina et al. (2001)

b Stoll (1980)

c Koponen et al. (1996)
}

\section{Appendix A. Biot Model for Porous Media}

Fast $\mathrm{P}$-wave velocity $\left(V_{\mathrm{P}}\right)$ is calculated by the following formulations:

$$
\begin{aligned}
& \left(\frac{H}{V_{\mathrm{p}}^{* 2}}-\rho_{\mathrm{mix}}\right) \cdot\left(q-\frac{I}{V_{\mathrm{p}}^{* 2}}\right)-\left(\frac{C}{V_{\mathrm{p}}^{* 2}}-\rho_{f}\right) \cdot\left(\rho_{f}-\frac{C}{V_{\mathrm{p}}^{* 2}}\right) \\
& =0, \text { and } \\
& V_{\mathrm{p}}=\operatorname{Re}\left(V_{\mathrm{p}}^{*}\right),
\end{aligned}
$$

where $V_{\mathrm{p}}^{*}$ is the complex $\mathrm{P}$-wave velocity, and $\operatorname{Re}\left(V_{\mathrm{p}}^{*}\right)$ is the real part of the complex $\mathrm{P}$-wave velocity. The parameters $A, I, H, C, F$, and $q$ are defined as follows:
$A=B_{\mathrm{g}} \cdot\left(1+n \cdot\left(\frac{B_{\mathrm{g}}}{B_{\mathrm{f}}}-1\right)\right)$,

$I=\frac{B_{\mathrm{g}}^{2}}{A-B_{\mathrm{sk}}}$,

$H=B_{\mathrm{sk}}+\frac{4}{3} \cdot G_{\mathrm{sk}}+\frac{\left(B_{\mathrm{g}}-B_{\mathrm{sk}}\right)^{2}}{A-B_{\mathrm{sk}}}$,

$C=\frac{B_{\mathrm{g}}-B_{\mathrm{sk}}}{A-B_{\mathrm{sk}}} \cdot B_{\mathrm{g}}$

$F=\frac{\zeta \cdot T}{4 \cdot\left(1+\frac{2 \cdot j \cdot T}{\zeta}\right)}$, and

$q=\frac{\alpha \cdot \rho_{f}}{n}-j \cdot \frac{\eta \cdot F}{\omega \cdot K}$

Attenuation $(1 / Q)$ is expressed as follows:

$\frac{1}{Q}=\frac{\operatorname{Im}\left(M^{*}\right)}{\operatorname{Re}\left(M^{*}\right)}$,

where $Q$ is the quality factor, and $M^{*}$ is the complex constraint modulus. $\operatorname{Im}\left(M^{*}\right)$ means the imaginary part, and $\operatorname{Re}\left(M^{*}\right)$ describes the real part of the complex constraint modulus (Table 3).

\section{References}

Biot MA (1956a) Theory of propagation of elastic wave in a fluidsaturated porous solid: I. Low-frequency range. J Acoust Soc Am 28(2):168-178

Biot MA (1956b) Theory of propagation of elastic wave in a fluidsaturated porous solid: II. Higher frequency range. J Acoust Soc Am 28(2):179-191

Brown ET (1981) Rock characterization testing \& monitoring. Pergamon Press, Oxford

Cadoret T, Mavko G, Zinszner B (1998) Fluid distribution effect on sonic attenuation in partially saturated limestones. Geophysics 63(1): $154-160$

Cha MS, Cho GC (2007) Compression wave velocity of cylindrical rock specimens: engineering modulus interpretation. Jpn J Appl Phys 46(7B):4497-4499

Gardner GHF, Wyllie MRJ, Droschak DM (1964) Effects of pressure and fluid saturation on the attenuation of elastic waves in sands. J Petr Tech 16(2):189-198

Johnston DH, Toksőz MN, Timur A (1979) Attenuation of seismic waves in dry and saturated rocks: II. Mechanisms. Geophysics 44(4):691-711

Kim DS, Kweon GC, Lee KH (1997) Alternative method of determining resilient modulus of compacted subgrade soils using free-free resonant column test. Transport Res Rec 1577: $62-69$

Koponen A, Kataja M, Timonen J (1996) Tortuous flow in porous media. Phys Rev E 54(1):406-410

Mavko GM, Nur A (1979) Wave attenuation in partially saturated rocks. Geophysics 44(2):161-178

Murphy WF (1982) Effects of partial water saturation on attenuation in Massilon sandstone and Vycor porous glass. J Acoust Soc Am 71(6):1458-1467 
Santamarina JC, Klein KA, Fam MA (2001) Soils and waves. Wiley, New York

Stoll RD (1980) Theoretical aspects of sound transmission in sediments. J Acoust Soc Am 68(5):1341-1349

Toksőz MN, Johnston DH, Timur A (1979) Attenuation of seismic waves in dry and saturated rocks: I. Laboratory measurements. Geophysics 44(4):681-690

Vaghela JG, Stokoe KH (1995) Small-strain dynamic properties of dry sand from the free-free resonant column. Geotechnical engineering report GT95-1, Geotechnical Engineering Center, University of Texas at Austin, Austin

Winkler KW, Nur A (1982) Seismic attenuation: effects of pore fluids and frictional sliding. Geophysics 46(1):1-15 